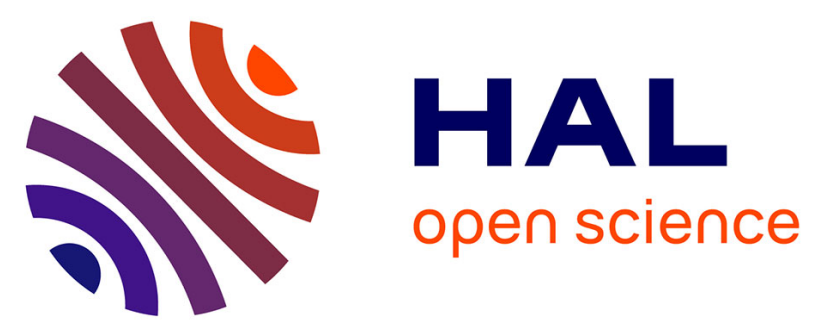

\title{
Coexistence between the future aeronautical system for continental communication L-DACS and the Distance Measuring Equipment DME
}

Najett Neji, Raul de Lacerda, Alain Azoulay, Thierry Letertre, Olivier Outtier

\section{- To cite this version:}

Najett Neji, Raul de Lacerda, Alain Azoulay, Thierry Letertre, Olivier Outtier. Coexistence between the future aeronautical system for continental communication L-DACS and the Distance Measuring Equipment DME. 2012 IEEE First AESS European Conference on Satellite Telecommunications (ESTEL), Oct 2012, Rome, Italy. pp.1 - 7, 10.1109/ESTEL.2012.6400087 . hal-00779141

\section{HAL Id: hal-00779141}

https://hal-centralesupelec.archives-ouvertes.fr/hal-00779141

Submitted on 21 Jan 2013

HAL is a multi-disciplinary open access archive for the deposit and dissemination of scientific research documents, whether they are published or not. The documents may come from teaching and research institutions in France or abroad, or from public or private research centers.
L'archive ouverte pluridisciplinaire HAL, est destinée au dépôt et à la diffusion de documents scientifiques de niveau recherche, publiés ou non, émanant des établissements d'enseignement et de recherche français ou étrangers, des laboratoires publics ou privés. 


\section{Coexistence between the future aeronautical system for continental communication L-DACS and the Distance Measuring Equipment DME}

\author{
Najett Neji, Raul de Lacerda, Alain Azoulay \\ SUPÉLEC - DRE \\ 91192 Gif Sur Yvette - France \\ \{surname.name\}@supelec.fr
}

\author{
Thierry Letertre \\ SUPÉLEC - TELECOM \\ 91192 Gif Sur Yvette - France \\ thierry.letertre@supelec.fr
}

\author{
Olivier Outtier \\ DGAC - DSNA \\ 75015 Paris - France \\ olivier.outtier@aviation-civile.gouv.fr
}

\begin{abstract}
In the last decade, the aeronautical authorities expressed their need to develop a new system for aeronautical radiocommunications. The L-band Digital Aeronautical Communication System (L-DACS) is the part of the future system that will be operating in a part of the aeronautical L-band $(960-1164 \mathrm{M} \mathrm{Hz})$, already occupied by a large number of radio-frequency legacy systems. This paper aims at studying the L-DACS impact on a legacy system, the Distance Measuring Equipment (DME), under two critical situations. Such topics are fundamental in aeronautics, as any communication or radionavigation dysfunction may endanger flight and passengers security. Some obtained results will be used for the L-DACS standardization and its specifications finalization. For the first scenario (air-to-air scenario), we propose a deterministic algorithm to compute the interference level in the frequency domain. Since this seems to be insufficient for the second scenario (co-site scenario), we develop a time-frequency approach to analyze the interference using an aeronautical RFC test-bed that we implemented at Supelec.
\end{abstract}

\section{INTRODUCTION}

In the beginning of the 21th century, international aeronautical authorities expressed their need to develop a new infrastructure for aeronautical radiocommunications, because the air-traffic is increasing (see Fig. 1) and that current communication systems in the aeronautical VHF-band $(118-136 \mathrm{MHz})$ between pilots and air-controllers are reaching their capacity limits. [1]

The L-band Digital Aeronautical Communication System (L-DACS) is the part of the future infrastructure that will be in charge of continental radiocommunication and it is expected to operate in a part of the aeronautical L-band (960 - $1164 \mathrm{MHz}$ ), already occupied by a large number of radio-frequency legacy systems (see Fig. 2). Among them, one of the most important systems is the Distance Measuring Equipment (DME), which permanently evaluates the slant distance between the aircraft and ground beacons, and which uses most of the $960-1164 \mathrm{M} \mathrm{Hz}$ spectrum.

Consequently, it is essential to consider its radio-frequency compatibility (RFC) for the development of the future L-DACS system. Through RFC, the coexistence between L-DACS and legacy systems can be evaluated, both in ground and airborne environments. L-DACS and a legacy system are considered compatible from a RFC perspective, if L-DACS can operate correctly (with respect to its expected quality of service) in the presence of the legacy system without generating harmful interference, and vice versa. To evaluate the RFC, it is essential ti study many interference scenarios, most of them are emphasized in Fig. 3.

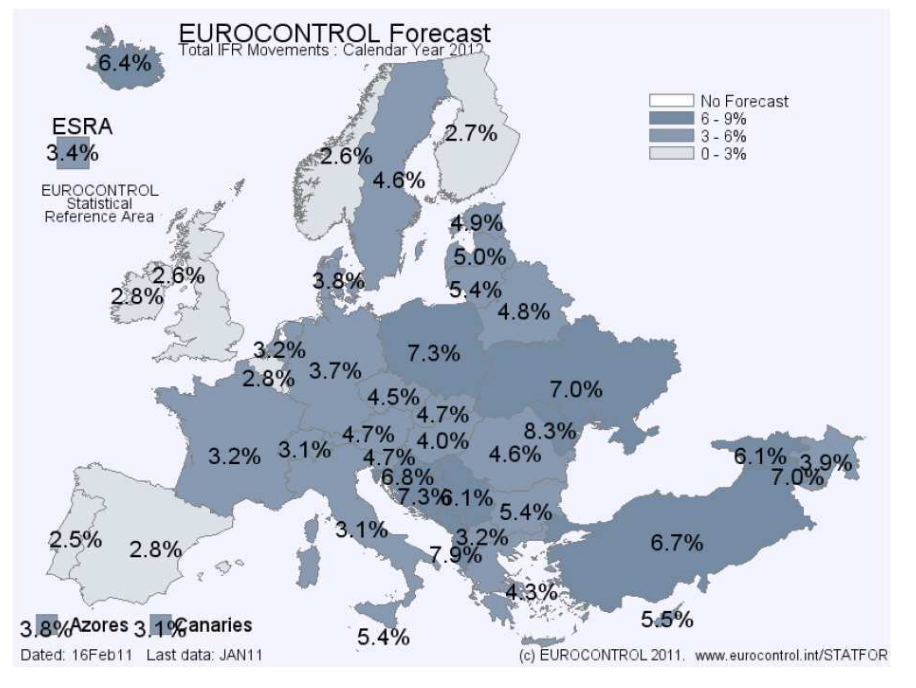

Fig. 1. Air traffic forecast in Europe for 2012 (from [2]).

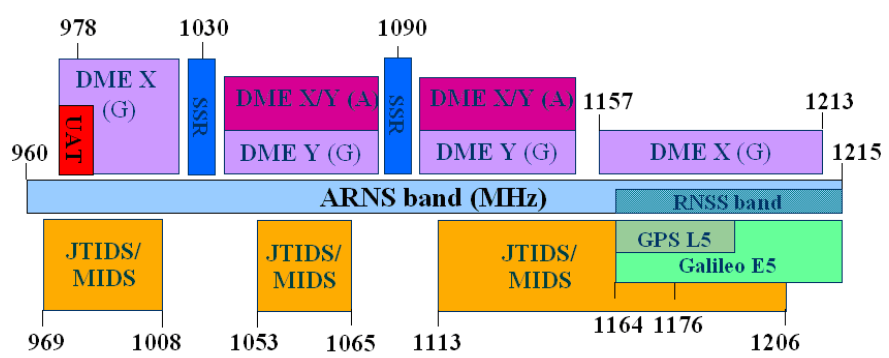

Fig. 2. The L-Band spectral occupancy (adapted from [3])

For the moment, two candidate technologies have been preselected and they are named L-DACS1 and L-DACS2, respectively. In this paper, we particularly focus on the L-DACS1/2 effect on the performance of a DME on-board receiver. To this end, we identified two among the most critical interference scenarios from the RFC viewpoint. Under the first scenario called the air-to-air scenario, L-DACS and DME are airborne equipment onboard of distinct aircrafts whereas for the second scenario, named the co-site scenario, both airborne devices are placed in the same aircraft.

The paper is organized as follows. In Section II, we give 


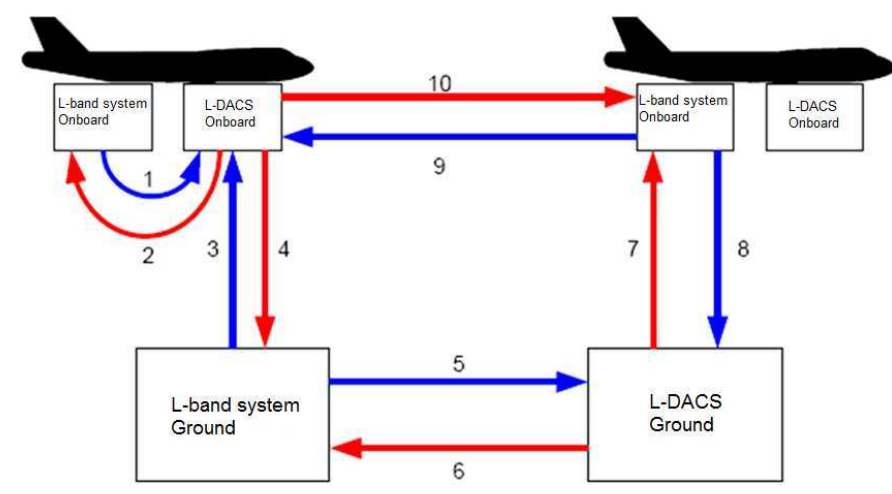

Fig. 3. Main interference scenarios to be studied for the RFC.

better insights on the L-DACS system and development status, and we present the DME system. In Sections III and IV, we analyze L-DACS impact on DME under the air-to-air scenario and co-site scenario, respectively. For both cases, we describe our methodology and emphasize the main results. Finally, in Section V, we summarize the paper contributions and we provide some perspectives for further research works.

\section{L-DACS AND DME SYSTEMS PRESENTATION}

Since their standardization, aeronautical communications are essentially analogical, and they are performed in the aeronautical VHF band $(118-136 \mathrm{MHz})$. On the other hand, according to the aeronautical authorities forecasts, the aerial traffic is expected to grow continually. Consequently, a congestion phenomenon is likely to happen in the few coming years in regions of the world with the highest traffic load.

In this context, the International Civil Aviation Organization (ICAO) launched in 2004 a Euro-American initiative to develop a future infrastructure for aeronautical communications (FCI). The FCI development started under a cooperative research program named Action-Plan 17 (AP17) [4] and involves research teams, industrial partners and aeronautical authorities from many countries in the world. The first step of the FCI development activities was to determine the most promising technologies to fulfill the new aeronautical requirements expressed in the report [5]. According to the first assessment results published in 2007 [6], [7], the infrastructure comprises many technologies depending on the flight phase.

Among these technologies, the L-band Digital Aeronautical Communication System (L-DACS) was identified to be in charge of the continental communications. It is expected to operate in a part of the aeronautical L-Band $(960-1164$ $\mathrm{MHz}$ ) [8]. The L-band is potentially large but its spectrum occupation is dense, not only by diverse aeronautical systems but also by mobile telephony and satellite navigation systems as it is shown in Fig. 2. Therefore, it is important to address the L-DACS coexistence with all these systems in both airborne and ground environments.

For the moment, two technologies have been identified as potential candidates to support L-DACS and they are named L-DACS1 [9], [10] and L-DACS2 [11], [12] . L-DACS1 is similar to the IEEE 802.16 wireless system and is based on Frequency-Duplex Division (FDD) technique, where the ground station and the airborne equipment transmit

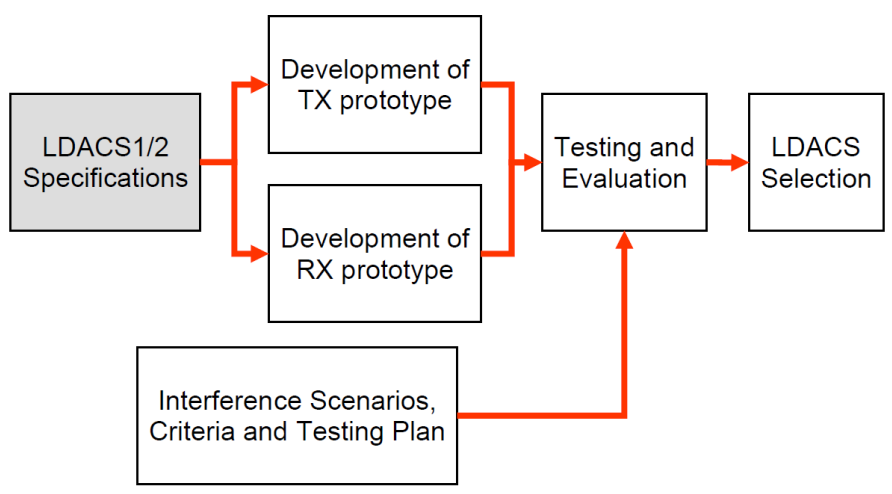

Fig. 4. The applied process for L-DACS candidate selection (from [9] and [11].

simultaneously using distinct frequency bands. However, the L-DACS2 is similar to the GSM standard and is based on Time-Duplex Division (TDD) technique, where the ground station and the airborne equipment transmit using the same carrier frequency during distinct time intervals. We summarize in Table ?? main system parameters of both candidates.

TABLE I

L-DACS1 AND L-DACS2 MAIN SYSTEM PARAMETERS [9]-[12]

\begin{tabular}{|c|c|c|}
\hline System Parameters & L-DACS1 & L-DACS 2 \\
\hline System range & $200 \mathrm{NM}$ & $200 \mathrm{NM}$ \\
\hline Airborne cable loss & $3 \mathrm{dBi}$ & $3 \mathrm{dBi}$ \\
\hline Transmitting effective bandwidth & $498,05 \mathrm{kHz}$ & $200 \mathrm{kHz}$ \\
\hline Maximum ground transmit power & $46 \mathrm{dBm}$ & $55,4 \mathrm{dBm}$ \\
\hline Maximum airborne transmit power & $46 \mathrm{dBm}$ & $47 \mathrm{dBm}$ \\
\hline Ground cable insertion losses & $2 \mathrm{~dB}$ & $2.5 \mathrm{~dB}$ \\
\hline Receiving effective bandwidth & $498,05 \mathrm{kHz}$ & $200 \mathrm{kHz}$ \\
\hline
\end{tabular}

L-DACS1/2 systems development is now a part of two research programs (SESAR [13] in Europe and NextGen [14] in the USA, additionally to parallel activities in Japan [15]) and three main tasks have been identified: systems specifications, prototypes development and performance evaluation through operational scenarios and interference scenarios. These tasks are shown in Fig. 4.

In this paper, we are interested in the third task and we aim to analyze the L-DACS1/2 impact on an L-band legacy system, the Distance Measuring Equipment (DME).

The DME [16] is a radio navigation system that is nowadays used in all airplanes to measure permanently the slant distance between an airplane and ground beacons. It operates in the 960-1215 $\mathrm{M} \mathrm{Hz}$ frequency band and has been used for near a century. The frequency channel used by the airplane and the one employed by the ground beacon are separated by $63 \mathrm{MHz}$. The DME communication is based on three steps. It starts on the airborne equipment (named the interrogator) which sends a stream of Gaussian-shaped pulse pairs to the ground station (called the transponder). The latter sends back the received signal to the interrogator with a certain delay, and finally, by measuring the time interval between sent and received streams, the interrogator determines its distance to the transponder.

According to its specifications, the DME transmitted signal consists of a maximum of 150 random pulse pairs per second. 


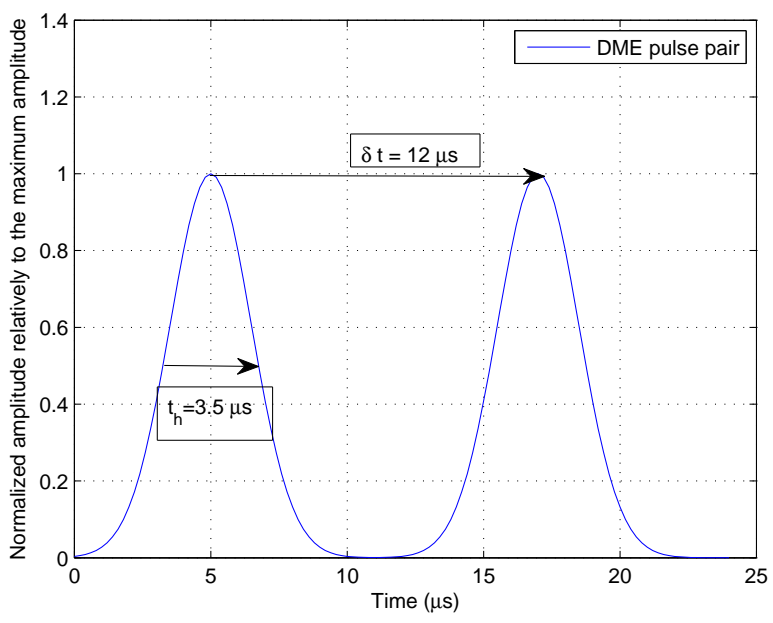

Fig. 5. The structure of a DME pulse pair

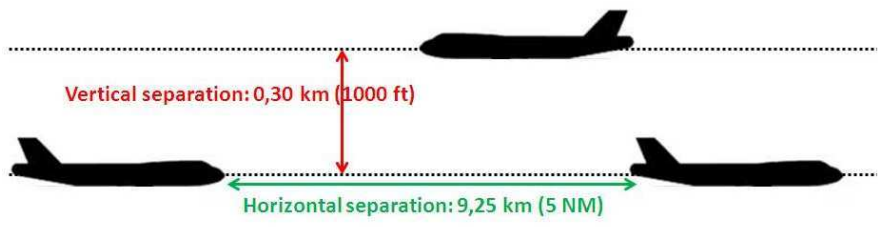

Fig. 6. Horizontal and vertical aircrafts separations imposed by aeronautical instances.

In each pair, the peaks of the two gaussian pulses are separated by at least $12 \mu \mathrm{s}$ depending on the DME mode of operation. A DME shape is given in Fig.

\section{AIR-TO-AIR INTERFERENCE SCENARIO ANALYSIS}

Under the air-to-air scenario, L-DACS and DME systems are onboard distinct aircrafts. This scenario is critical because the victim receiver is likely to intercept signals from many interferers (this is due to its high radio visibility). Moreover, this scenario seems useful for both the aeronautical network dimensioning and frequency/distance planning.

We evaluated the L-DACS1/2 interference effect based on a frequency-domain analysis and using a specific algorithm to compute the interference level in the worst case. Under this situation, the victim receiver is likely to get the maximum interference level. In addition, we considered the case of co-channel interference, where the interferer and the victim receiver use overlapping frequency bands. Finally, we assumed a free-space propagation model.

We defined a deterministic approach, different from existing methodologies dealing with L-DACS interference, taking into account the imposed vertical and horizontal aircrafts separations by aeronautical authorities (see Fig. 6).

To model the aeronautical environment, we used L-DACS antennas characteristics (which are typical L-band antennas,

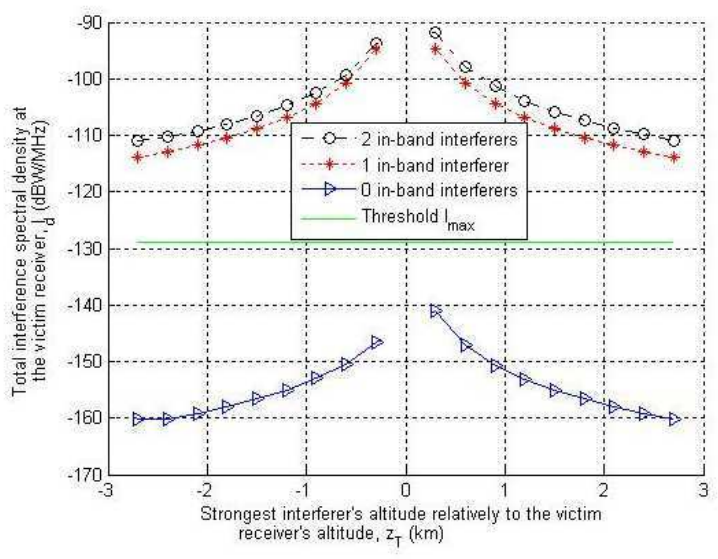

Fig. 7. Total interference spectral density at the DME victim receiver, by two L-DACS1 transmitters (for 2, 1 and 0 in-band interferers).

omnidirectional in azimuthal direction and slightly directive in vertical direction to place interferers in a bi-dimensional grid. The methodology assumes at maximum two airplanes per flight level and is based on three steps. In the first step, we identify the strongest one/two interferer(s) for each flight level independently. In the second step, we select the positions of the $K$ identified airplanes generating the highest cumulative interference power at the victim receiver. In the last step, we allocate to each interferer a frequency channel such as the interference level remains the highest.

In previous publications [18] and [19], we applied this approach to an ideal victim receiver. In this paper, we analyze the L-DACS interference considering a DME receiver.

From their specifications, L-DACS1 uses a $0.5 \mathrm{MHz}$ bandwidth for transmission/reception and its airborne transmission power is $16 \mathrm{dBW}(46 \mathrm{dBm})$; and L-DACS2 uses a $0.2 \mathrm{MHz}$ bandwidth for transmission/reception and its airborne transmission power is $17 \mathrm{dBW}(47 \mathrm{dBm})$. The DME uses a $1 \mathrm{MHz}$ bandwidth and for the DME receiver, the computed maximum acceptable interference spectral density is $-129 \mathrm{dBW} / \mathrm{MHz}(-99 \mathrm{dBm} / \mathrm{MHz})$. It should be noticed that aeronautical safety margins have to be added to obtain the operational acceptable threshold.

Based on these information, we considered $K=2$ L-DACS interferers around the DME victim receiver. Three cases may occur: both interferers are in-band, or only one of them is in-band or both of them are out-of-band. We computed the cumulative interference spectral density in the three situations and the results are summarized in Fig. 7 and Fig. 8. In these figures, we present the total interference spectral density $I_{d}$ at the DME receiver, with respect to vertical separation between the strongest L-DACS interferer and the DME receiver altitudes.

From these results, we can see that as expected by classical frequency sharing studies, it is necessary to have no in-band L-DACS1/2 interferers in order to avoid harmful interference. We also notice that interference is principally due to the 


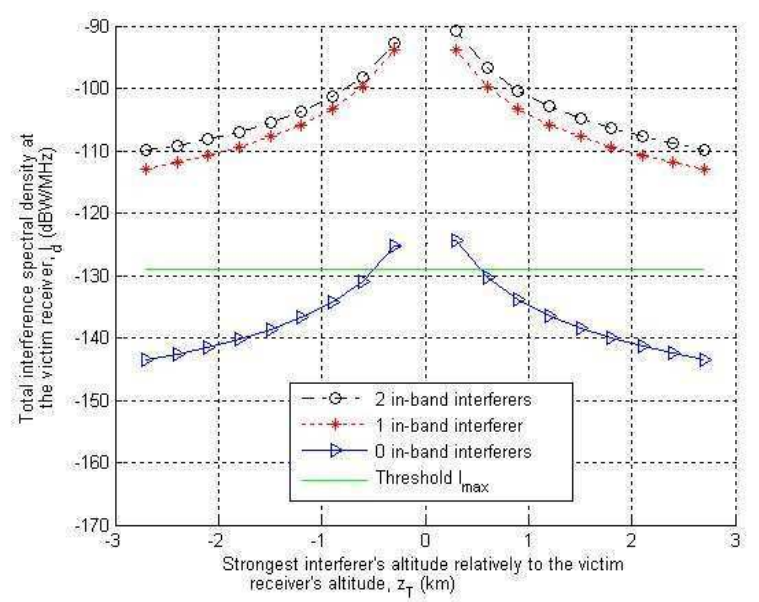

Fig. 8. Total interference spectral density at the DME victim receiver, by two L-DACS2 transmitters (for 2, 1 and 0 in-band interferers).

first identified L-DACS interferer (which is the strongest interferer). Moreover, for both L-DACS candidates, we obtained a precise estimation of the interference level using our deterministic approach.

\section{CO-SITE INTERFERENCE SCENARIO ANALYSIS}

For the second identified interference scenario, called the co-site scenario, both the interferer and the victim receiver are on-board the same aircraft. This scenario is considered as the most critical from a RFC viewpoint because of equipment proximity. From now on, we precisely focus on one of the L-DACS candidates on the DME performance and we chose to investigate L-DACS2.

The frequency-domain analysis seems insufficient for this case. In fact, the interference level $P_{D M E}$ received by the DME interrogator is given by equation (1):

$$
\begin{gathered}
P_{D M E}(d B W)=P_{L D A C S 2}(d B W)-L_{L D A C S 2}(d B) \\
-C_{A}(d B)-L_{D M E}(d B)+M_{d B}(d B)
\end{gathered}
$$

where $P_{L D A C S 2}$ (in $d B W$ ) is the L-DACS2 output power, $L_{L D A C S 2}$ and $L_{D M E}$ (in $d B$ ) are cable losses at the LDACS2 transmitter and the DME interrogator, respectively, and $C_{A}$ (in $d B$ ) is the coupling between their antennas. $M_{d B}$ (in $d B$ ) is the attenuation obtained by the superposition between the L-DACS2 transmit mask and the DME blocking mask.

Now, using system characteristics given in [11], we have $P_{L D A C S 2}=17 \mathrm{dBW}$ and $L_{L D A C S 2}=3 \mathrm{~dB}$. In addition, according to [20], $C_{A}=20 \mathrm{~dB}$. Moreover, at $1 \mathrm{MHz}$ offset relatively to its center frequency, the L-DACS2 maximum mask attenuation is $80 d B$ (see [21]). Hence, if L-DACS2 and DME frequencies separated by $1 \mathrm{MHz}, M_{d B}=-80 \mathrm{~dB}$. If we assume that $L_{D M E}=2 d B$, we obtain $P_{D M E}=-88$ $d B W$. Knowing that the DME bandpass is equal to $1 \mathrm{MHz}$, the interference density is $I_{D M E}=-88 \mathrm{dBW} / \mathrm{MHz}$ at the DME receiver interrogator input.

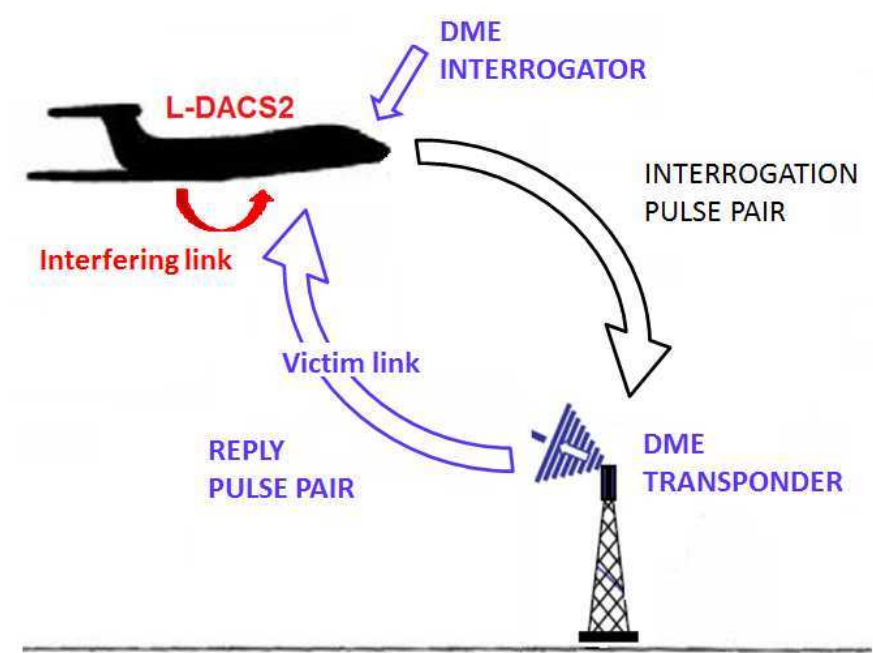

Fig. 9. RFC system model: DME transmitter, L-DACS2 interferer and DME victim.

However, as mentioned in [22], the maximum acceptable interference level by the DME interrogator is $I_{\max }=-129$ $d B W / M H z$ (before taking into account aeronautical margins).

Thus, to study this scenario, we consider a different methodology, named time-domain approach, considering system dynamics and technology properties. Let us first describe the interference process in this case (see Fig. 9).

The L-DACS interference is added to the DME signal sent by the transponder to the interrogator (the reply signal, which is a replica of the interrogation signal). In this situation, collision between L-DACS and DME signals may occur. Due to this phenomenon, some Gaussian-pulse pairs may be lost at the DME airborne receiver. Let us call $R$ (in \%) the rate of correctly received DME pairs, compared to the total number of sent pairs in the interrogation signal. If $R$ becomes lower than a certain threshold Rmin after a precise time delay (called the maximum synchronization time), the synchronization between the DME interrogator and the DME ground beacon is considered lost.

In this paper, we implement the scenario considering an L-DACS2 interferer (TDD system), through specific test-beds with an L-DACS2 signal generator and DME commercial equipment. Our criterion of analysis is the DME synchronization state (binary information), which can be easily detected by observing the DME interrogator screen during the maximum synchronization time (see Fig. 10). The synchronization state is 1 if the distance is displayed with acceptable precision (referring to DME interrogator specifications) before the maximum synchronization time expires, and 0 else.

In the previous papers [23] and [24], we presented the obtained measurement results for the conducted mode, where L-DACS unwanted signals are propagated through cables, connectors and electronic devices. In this work, we are more focused on laboratory measurements in the radiated mode, where unwanted signals are radiated by the L-DACS2 antenna and captured by the DME antenna. For this mode, we first enumerate the used material, then we describe the experimental setup and we detail the followed protocol. 


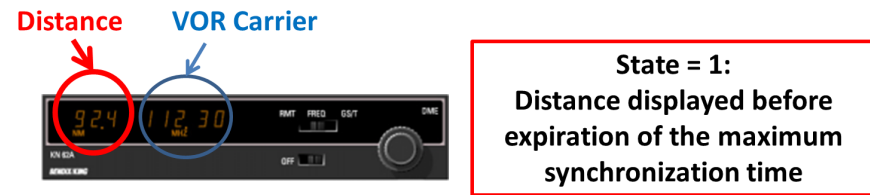

Fig. 10. Displayed information by the synchronized DME interrogator.

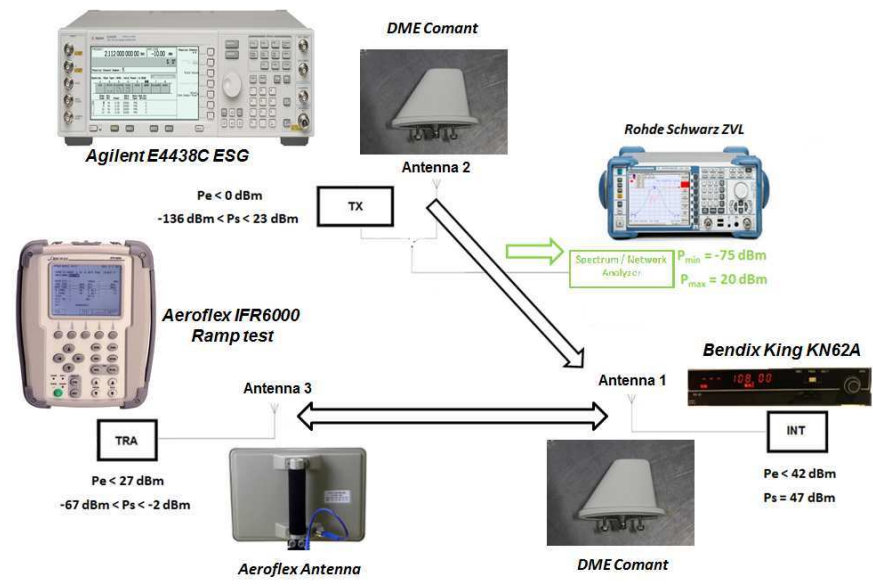

Fig. 11. Schematic diagram for RFC measurements in the radiated mode.

For the experimental material (see Fig. 11), we associate an antenna to the L-DACS2 transmitter, an antenna to the DME interrogator and an antenna to DME transponder. In addition to the L-DACS2 signal generator (Agilent E4438C ESG), the DME used commercial interrogator (Bendix King KN62A) and the DME commercial transponder (Aeroflex IFR6000 Ramp test), the test-bed comprises:

- Two identical commercial aeronautical antennas manufactured by "Comant" and associated to the DME interrogator (antenna 1) and the L-DACS2 generator (antenna 2),

- A specific antenna provided with the Aeroflex IFR6000 pack and associated to the DME transponder (antenna 3),

- A network/spectrum analyzer manufactured by "Rohde and Schwarz" (ZVL type) to measure signals at antenna ports of the different devices.

In Fig. 11, black-colored arrows indicate the power circulation starting from the DME interrogator and green-colored arrows show outputs to spectrum analyzers. We also precise the minimum and maximum output powers $P_{s}$ and maximum input power $P_{e}$ of our equipments as well as the minimum and maximum input power of our spectrum analyzers. We implemented this diagram with commercial equipments and antennas at the planar anechoic chamber at Suplec (see Fig. 12).

To perform the measurements, we proposed the following experimental protocol, based on the reference document [20], and composed by 11 steps ${ }^{1}$ :

1 - Verify the link budget before beginning measurements.

${ }^{1}$ For a fixed power in step 7, repeat steps 8 to 11 until the DME synchronization state changes. Then, increase the power and follow the same procedure.
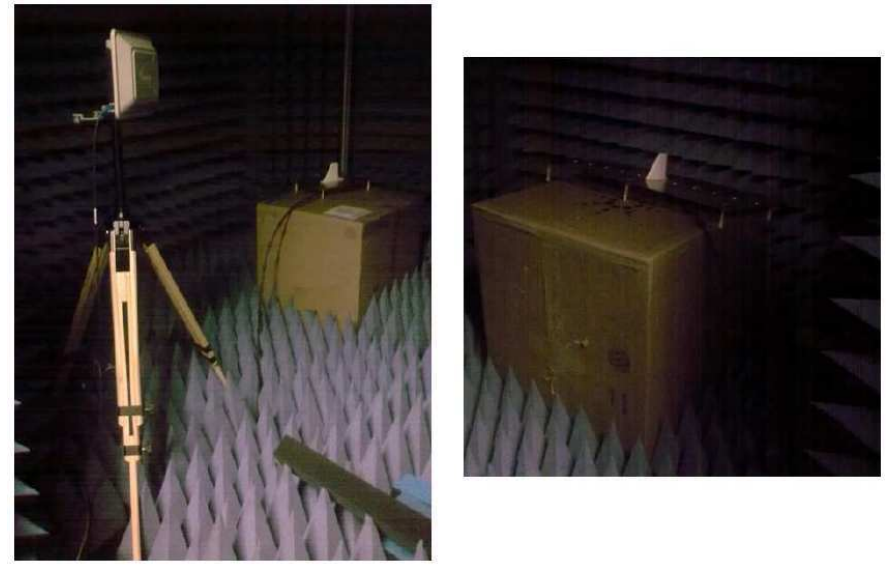

Fig. 12. Implemented aeronautical RFC test-bed in the radiated mode.

2- Switch on the DME interrogator's alimentation.

3- Turn on the DME interrogator and set its VOR frequency (based on tables from [20], this selected VOR frequency will determine a specific DME channel and consequently both interrogator and transponder center frequencies).

4- Check L-DACS2 and DME equipments' good functioning through preliminary tests.

5- Generate the L-DACS2 time-domain radio-frequency (RF) signal.

6- Activate the L-DACS2 generator "local" mode.

7- Set the L-DACS2 maximum power (depending on the target $S I R$ value), its modulation and set its center frequency.

8- Increase the L-DACS2 channel occupation rate.

9- Run the test.

10- Count a certain time delay (corresponding to the maximum synchronization time) and observe the DME synchronization state. For each measure, we verify both stability and precision criteria. We considered that a measure is stable if the same DME synchronization state is obtained after two consecutive tests under the same conditions.

\section{1- Stop the test.}

We also assumed in-band interference and out-of-band interference. For the experimental protocol, we considered 2 seconds for the maximal synchronization time, and $S_{\text {min }}+3$ $d B$ for the useful signal power at the DME victim receiver, being $S_{\min }$ (in $d B$ ) the tested DME equipment sensitivity (both values are based on the DME specifications). Finally, we selected the DME channel $17 X$, which corresponds to the carrier frequency $F_{c}=978 \mathrm{MHz}$ for the transponder (this corresponds to the lowest operational frequency for the investigated DME device). Under these assumptions, we analyze the impact of the L-DACS2 channel occupation rate, which is the percentage of time of effective L-DACS2 


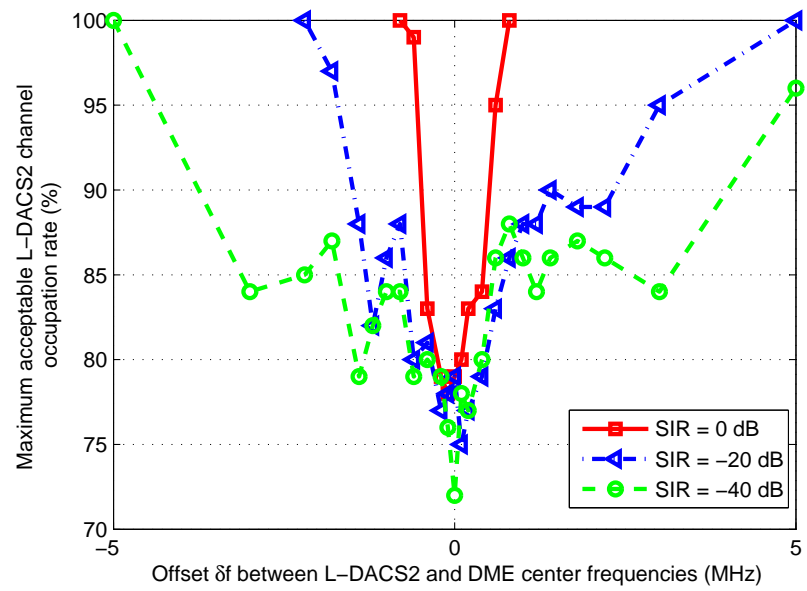

Fig. 13. DME receiver performance measurement in presence of L-DACS2 interference, with respect to the frequency offset between useful and interfering transmitters

transmission, on the DME performance. We summarize the obtained results in Fig. 13, where the maximum acceptable L-DACS2 channel occupation rate is shown with respect to the frequency separation between L-DACS2 and DME transponder signal carriers, for different values of the Signal to Interference Ratio (SIR) at the DME airborne receiver.

We can see that under this scenario and with the specific tested equipment, the DME synchronization is successful in the presence of the L-DACS2 interferer if its channel occupation rate remains lower than $72 \%$ for $S I R$ higher than $-40 d B$ and this also occurs in the co-channel mode (L-DACS2 and DME use overlapping channels). Hence, based on our proposed approach, implemented test bed and available DME commercial equipment at Supelec, L-DACS2 would not cause harmful interference on the DME system. We prove herein that while time-domain parameters are considered, satisfying results in terms of RFC can be obtained. In fact, according to existing RFC studies, in the co-channel mode, these systems cannot coexist.

\section{CONCLUSION}

In this paper, we studied the L-DACS interference impact on the DME receiver performance under some critical scenario from the RFC viewpoint (air-to-air scenario and co-site scenario). To analyze the first scenario, we proposed a deterministic frequency-domain methodology which is different from existing approaches mentioned in frequency sharing studies involving L-DACS, and which gives a good estimation of the interference level at the victim receiver. For the second scenario we developed a time-frequency approach, which takes into account system dynamics and which provides more realistic evaluation to the actual interference level. More particularly, for the co-site scenario, we proved that under this new methodology and using a specific laboratory setup to emulate the interference environment, L-DACS2 would not cause harmful interference on the DME interrogator, whereas in classical studies, this was not expected to happen. For further work, these results should be confirmed by additional tests with other commercial DME devices using other metrics to analyze their performance. To complete the analysis, it is also important to study the DME effect on the L-DACS2 performance using the time-frequency approach, then to extend it to other interference scenarios, in order to evaluate the interference more precisely than under classical RFC methodologies. We finally suggest generalizing the proposed time-frequency approach to analyze the RFC between any two radio-frequency systems, taking into account additional parameters related to system dynamics and their technology properties.

\section{REFERENCES}

[1] J. Hakegard, "Spectral Efficient COMmunications for future Aeronautical Services (SECOMAS)," in VERDIKT-conference Hell, October 2007.

[2] Medium Term Forecast - Flight Movements 2011-2017, EUROCONTROL, February 2011.

[3] Electronic Communications Committee (ECC) - European Conference of Postal and Telecommunications Administrations (CEPT), "Proposed amendments to the Working Document towards a Preliminary draft new report ITU-R M. on AM(R)S sharing feasibility in the $960-1164 \mathrm{MHz}$ band," 2008, doc. ECC/CPG11/PTC(2008)45 - Annex 04.

[4] FAA/EUROCONTROL COOPERATIVE R\&D, "Action Plan 17 : Future Communication Study - Terms of reference," 2004, doc. Reference Ap1704-TOR-V1.0.

[5] Future Communication Study - Operational Concepts and Requirements Team, "Communications Operating Concept and Requirements for the Future Radio System,” EUROCONTROL/FAA, Tech. Rep., 2007, version 2.0 .

[6] M. Schnell, "Future Communications Infrastructure - Step 2: Technology Assessment Results - version 1.0," EUROCONTROL, Tech. Rep., 2007.

[7] T. Gilbert et al., "FCS Phase II - Identification of Technologies for Provision of Future Aeronautical Communications," NASA, Tech. Rep., 2006.

[8] European Conference of Postal and Telecommunications Administrations (CEPT), "World Radiocommunication Conference (WRC-12) - Plenary Meeting - European Common Proposals for the Work of the Conference - Part 4 - Agenda Item 1.4," CPG-12-7, Addendum 4 to Document 5-E, 2011.

[9] European Organization for the Safety of Air Navigation, "L-DACS1 System Definition Proposal: Delivrable D2," EUROCONTROL, Tech. Rep. Edition 1.0, 2009.

[10] "L-DACS1 System Definition Proposal: Delivrable D3 : Design Specifications for L-DACS1 Prototype," EUROCONTROL, Tech. Rep. Version 1.0, 2009.

[11] — "L-DACS2 System Definition Proposal: Delivrable D2," EUROCONTROL, Tech. Rep. Version 1.0, 2009.

[12] _ , "L-DACS2 Transmitter and Receiver prototype equipment specifications : Deliverable D3," EUROCONTROL, Tech. Rep. Version 1.2, 2009.

[13] SESAR Consortium, "SESAR Definition Phase - Delivrable D6 - Work Programme for 2008 - 2013," co-funded by the European Commission and EUROCONTROL, Tech. Rep., 2008, doc. Reference D L M - 0710 $-002-02-00$.

[14] Joint Planning and Development Office, "Concept of Operations for the Next Generation Air Transportation System," 2007, version 2.0.

[15] S. Nagaoka, "Long-term Vision of ENRI's Research and Development," Electronic Navigation Research Institute, Tech. Rep. Version.4, 2008 , translated from Japanese Text.

[16] International Civil Aviation Organization, Aeronautical Telecommunications - Annex 10 to the Convention on International Civil Aviation Volume 1: Radio Navigation Aids, 6th ed., amendment 84, July 2009.

[17] N. Neji et al., "Interference Analysis for the Future Aeronautical Communication System," in Personal Indoor Mobile Radiocommunications Conference, September 2009.

[18] _ "Radioelectric Compatibility of the Future Aeronautical Communication System," in International Conference on ITS Telecommunications, October 2009.

[19] European Organisation for Civil Aviation Equipment (EUROCAE), "Minimal Operational Performance Requirements for Distance Measuring Equipment Interrogator (DME/P and $\mathrm{DME} / \mathrm{N}$ ) operating within the Radio Frequency Range 960 to $1215 \mathrm{MHz}$ (Airborne Equipment)," January 1987, document ED-54.

[20] N. Neji, R. de Lacerda, A. Azoulay, T. Letertre, and O. Outtier, "Interference analysis for the future aeronautical communication system," in IEEE 20th International Symposium on Personal, Indoor and Mobile Radio Communications, sept. 2009, pp. $1236-1240$.

[21] International Telecommunication Union - Radiocommunication Sector, "Protection criterion for the aeronautical radionavigation service with respect to aggregate emissions from space stations in the radionavigationsatellite service in the band 1 164-1 $215 \mathrm{MHz}$," Recommendation ITU-R M. 1639-1, 2005.

[22] N. Neji et al., "Effect of the aeronautical L-DACS2 radio-frequency signals on the DME system performance," in IEEE Vehicular Technologies Conference, 2010.

[23] - "How the aeronautical L-DACS2 radio-frequency signals modulation affects the DME system performance," in IEEE Vehicular Technologies Conference, 2010 